\title{
Dual-Polarized 28-GHz Air-Filled SIW Phased Antenna Array for Next-Generation Cellular Systems
}

\author{
Kamil Yavuz Kapusuz, Student Member, IEEE, Sam Lemey, Member, IEEE, \\ and Hendrik Rogier, Senior Member, IEEE
}

\begin{abstract}
A high-performance dual-polarized eight-element air-filled substrate-integrated-waveguide (AFSIW) cavity-backed patch antenna array is presented. The antenna operates in the [26.5-29.5] $\mathrm{GHz}$ band and provides a stable high data-rate wireless communication link between end-user terminals and access points in next-generation cellular systems. Its topology is carefully selected to maximize the performance of the array. In addition, by combining the AFSIW technology with a new antenna architecture, a low-profile, low-cost, stable, and high-performance array design is guaranteed. A prototype was fabricated and validated, demonstrating a wide active impedance bandwidth over $\pm 3^{\circ}$ scanning range and low-cross polarization level within the entire frequency band.
\end{abstract}

Index Terms-Air-filled substrate-integrated-waveguide (AFSIW), aperture-coupled antenna, cavity-backed patch antenna, dual-polarized, $5 \mathrm{G}$ wireless communication, phased antenna array.

\section{INTRODUCTION}

$\mathbf{T}$ HE fifth-generation (5G) wireless communication systems will improve multiple key aspects of wireless communication to support the ever increasing demand for reliable and low-latency high-data rate mobile data traffic between end-user terminals and access points [1]. To address these stringent 5G goals, the next generation of wireless communication systems will exploit the large bandwidths available at millimeter wave (mmWave) bands [2], while adopting multi-antenna systems to leverage spatial multiplexing via highly directive communication links [3] and featuring ultra-dense deployment of smallcells [4].

In such a scenario, multiple short-range base stations (BSs) will be densely deployed using large-scale multi-antenna systems to precisely focus pencil-beams to end users. This will improve the cell border coverage and enhance the spectral efficiency and frequency reuse factor of the network. In addition, these pencil beams overcome the extremely challenging propagation conditions at the mmWave bands [5]. However, to achieve a broad market acceptance, these large-scale antenna systems need to be cost-effective, non-obstructive and lowweight. Moreover, they should provide beam scanning ability. Hence, there is a stringent need for innovative phased antenna

This work was supported in part by ERC through "ATTO: A new concept for ultrahigh capacity wireless networks" under Grant 695495 .

K.Y. Kapusuz, S. Lemey, and H. Rogier are with the Electromagnetics Group, Department of Information Technology, IDLab, Ghent UniversityIMEC, Technologiepark Zwijnaarde 15, Ghent B-9052, Belgium. (e-mail: kamilyavuz.kapusuz@ugent.be; sam.lemey@ugent.be; hendrik.rogier@ugent.be). array architectures that support adaptive steering of directive beams.

In this paper, a wideband dual-polarized eight-element airfilled substrate-integrated-waveguide (AFSIW) cavity-backed patch antenna array covering the [26.5-29.5] GHz 5G band is presented. By an AFSIW implementation of the cavity, a lowloss low-cost antenna is obtained with highly efficient stable radiation characteristics and excellent antenna/platform isolation. A wideband antenna performance is achieved by exciting two hybrid modes. To exploit polarization diversity for reliable and low-latency high-data rate mobile data traffic between enduser terminals and access points, the antenna array consists of eight dual-polarized antenna elements that are rotated by $45^{\circ}$. A $70^{\circ}$ scanning range for both polarizations is achieved. To the authors' best knowledge, this is the first paper that describes an innovative highly-efficient low-cost dual-polarized AFSIW phased antenna array structure capable of steering highly directive beam for $5 \mathrm{G}$ cellular systems.

In recent years, multiple phased antenna arrays [6]-[9] have been proposed for $5 \mathrm{G}$ wireless communication systems. A single-polarized $4 \times 8$ antenna array composed of two $4 \times 4$ multilayer arrays is presented in [6]. About $25^{\circ}$ beam steering is achieved in a bandwidth of [22 - 32] $\mathrm{GHz}$ with a gain of approximately $19 \mathrm{dBi}$. In [7], a multi-beam antenna composed of four single-polarized 1 x 4 multilayer SIW slot antenna arrays is designed. The antenna array can produce sixteen predefined sector beams with a gain of $14.5 \mathrm{dBi}$ at $28 \mathrm{GHz}$. A single-polarized 64-element phased-array formed by a $2 \times 2$ transmit/receive beamformer chip is presented in [8] at $28 \mathrm{GHz}$. The phased array can scan up to $\pm 50^{\circ}$ in azimuth (H-plane) and $\pm 25^{\circ}$ in elevation (E-plane). An overview of some existing multibeam antenna technologies for $5 \mathrm{G}$ wireless communication is presented in [9].

This paper is organized as follows. In Section II, the topology and operation principle of the stand-alone antenna element is discussed. Moreover, the manufacturing process is explained in full detail, including a thorough corner analysis. Section III covers the design and construction of the $8 \times 1$ phased antenna array. In Section IV, a prototype of the antenna is validated. Conclusions and an outlook on future research are presented in Section V. 


\section{Stand-Alone AFSIW Antenna ElEment}

\section{A. Antenna Design Tradeoffs}

Large-scale highly-directive mmWave phased antenna arrays will play a key role in next-generation cellular mobile broadband communication systems. To guarantee peak performance of such a complex antenna system, the individual antenna element needs to be designed with utmost care and a challenging set of technical and economical requirements is imposed. Obviously, it should exhibit high and stable performance over a broad bandwidth in terms of radiation efficiency, half-power beamwidth and front-to-back ratio. Next, the proposed antenna topology needs to be compact to guarantee a broad gratinglobe free scanning range. To facilitate integration with active electronics, it must be low-profile, while providing high isolation between adjacent antenna elements. Moreover, fabrication cost and complexity have to be kept to the bare minimum to pave the way for deep market penetration. These tradeoffs make the design of an efficient mmWave-band antenna system without expensive multi-layer RF laminates and high-precision fabrication a real challenge.

The proposed wideband dual-linearly polarized aperturecoupled AFSIW cavity-backed patch antenna reconciles these conflicting demands in terms of performance, cost and footprint. In particular, following design requirements are imposed. The reflection coefficient with respect to $50 \Omega$ of each port should remain below $-10 \mathrm{~dB}$ in the entire [26.5-29.5] GHz band, while guaranteeing an intra-element port-to-port isolation larger than $25 \mathrm{~dB}$. In addition, the antenna element should exhibit a crosspolarization discrimination larger than $15 \mathrm{~dB}$, a front-to-back ratio larger than $15 \mathrm{~dB}$ and a half-power beamwidth of more than $70^{\circ}$ over the entire frequency band of operation. Finally, a radiation efficiency of more than $80 \%$ is required.

\section{B. Principle of Operation}

The proposed wideband dual-linearly polarized aperturecoupled AFSIW cavity-backed patch antenna is depicted in Fig. 1. A stacked AFSIW-based antenna technology is exploited to overcome the drawbacks of microstrip antennas (low efficiency and narrow bandwidth) while preserving the advantages of planar technology, being cost-effective fabrication, light weight and compact integration with monolithic microwave integrated components (MMICs), such as phase shifters and amplifiers. In addition, judiciously positioned rows of vias guarantee maximal suppresion of surface waves and excellent antenna/platform isolation.

The stacked AFSIW antenna is composed of three layers: the radiating layer (Layer 1), the cavity layer (Layer 2) and the feed layer (Layer 3). The thickness and dielectric properties of each substrate are carefully chosen to achieve the required impedance bandwidth and a very high efficiency, while maintaining a small footprint and keeping back radiation and cost low. To obtain a wide impedance bandwidth and high efficiency, a circular radiating patch is printed on a thin lowpermittivity substrate, forming Layer 1, and an air-filled cavity is introduced underneath the patch in Layer 2. To protect the patch from detrimental environmental effects, it is printed on the bottom side of its dielectric substrate. Next, the cavity

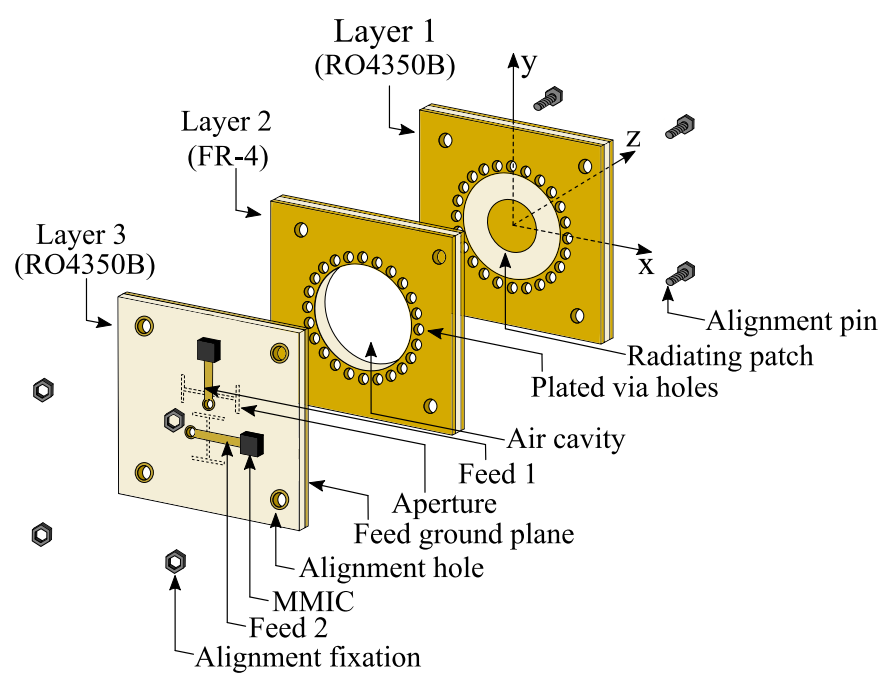

Fig. 1. Exploded view of the stand-alone AFSIW cavity-backed patch antenna element.

sidewalls are realized in Layer 2 by rows of plated via holes, at locations that are very close to the edges of the aircavity. This avoids that the electromagnetic fields penetrate substrate Layer 2 and minimizes mutual coupling between adjacent antenna elements. A small dielectric supporting region inside the cavity is preserved to guarantee sufficient mechanical strength. The design procedure described in [10] was leveraged to minimize loss and to obtain nearly substrate-independent behavior. As a result, a low-cost substrate material may be used to implement Layer 2 without compromising antenna performance. As shown in Fig. 1, the circular cavity-backed patch is fed by microstrip aperture coupling. Two perpendicular $\mathrm{H}$-shaped apertures are used to maximize bandwidth and to excite two orthogonal linear polarizations, which can be used to generate circular polarizations in future designs. Leveraging aperture coupling also guarantees shielding between the antenna and the feed network, and contributes to radiation pattern purity. Back radiation is minimized by implementing the feed network on a thin low-loss dielectric substrate (Layer 3) and exploiting short-ended microstrip lines to excite the $\mathrm{H}$-shaped apertures. Tuning is accomplished by trimming the short-circuited stub of the microstrip feed line and by adjusting the dimensions of the aperture. As shown in Fig. 1, MMICs can be compactly integrated on Layer 3 at the back of the antenna element.

The stand-alone antenna element was designed in two steps to ensure dual-linear polarization over a broad bandwidth. First, a single-feed single-polarized aperture-coupled AFSIW cavity-backed patch antenna topology was conceived such that two modes are excited at two neighbouring frequencies. The mode at the lower frequency combines a weak $T M_{010 \text {, cavity }}$

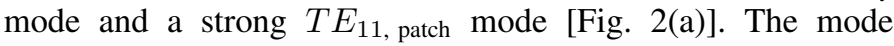
at the higher frequency consists of strong $T M_{010}$, cavity and

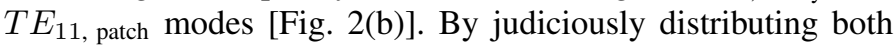
resonant frequencies over the frequency band of operation, the impedance bandwidth is increased significantly and the envisioned band is covered. To generate a second polarization, in the second and final step, a second feed line with $\mathrm{H}$-spaped aperture is inserted by rotating the first feed line and aperture, and shifting it along the axis, as shown in Fig. 1. To provide 


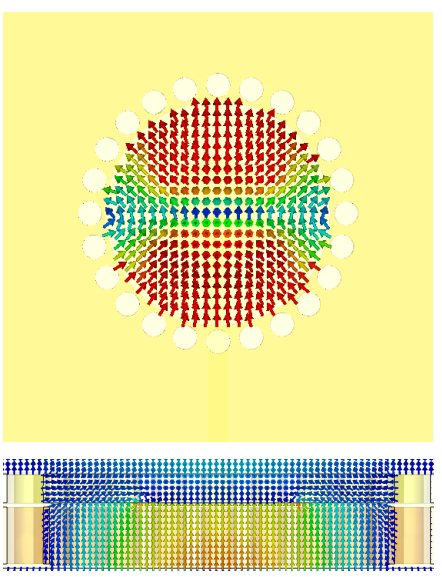

(a)

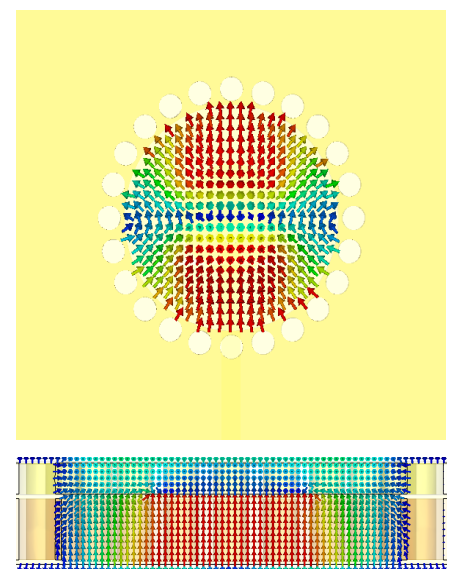

(b)

Fig. 2. Simulated hybrid mode profiles: electric field [V/m] at (a) $27.5 \mathrm{GHz}$ and (b) $29.8 \mathrm{GHz}$.

the same wideband performance for this second feed at second polarization state, the same modes are excited. At both resonant frequencies, a large electric field occurs across the antenna aperture, ensuring strong radiation in the hemisphere along the positive $z$-direction and weak radiation along the negative $z$-direction.

\section{Manufacturing Process}

The antenna has been optimized for high-performance mass production without using expensive multi-layer/thick RF PCB laminates. Therefore, in our design, only standard single layer PCB laminates were used to realize a cost-effective, broadband and modular solution. In particular, all layers (Layers 1-3) were fabricated by means of standard single layer PCB manufacturing technology at Eurocircuits [11]. The conductive parts of each layer were realized using a standard photolithography technique. A $0.508-\mathrm{mm}-\mathrm{RO} 4350 \mathrm{~B}$ substrate is exploited to construct Layer 1 and Layer 3 [ Fig. 1]. Layer 2 is implemented in lowcost 1-mm-FR4 substrate. The air-filled cavity is implemented by milling out a cylinder with diameter $2 b$, after which plated via holes (diameter $d$ ) are inserted around this air-filled region [Fig. 3(b)]. Finally, all layers are held together using 1-mmdiameter alignment pins and fixations [Fig. 1], to guarantee sufficient electrical contact and to provide correct alignment between layers. This allows a modular design, in which each part can be replaced later on with a different version.

Prior to the manufacturing, the antenna was optimized by using the frequency domain solver of CST Microwave Studio. Utmost care was taken to ensure that all design requirements, put forward in Section II.A, are met with sufficient margin, to account for variations in the material parameters and inaccuracies of the fabrication process. The antenna dimensions resulting from an extensive optimization process are shown in Fig. 3. The simulated magnitude of the reflection coefficients $\left[\left|S_{11}\right|\right.$ and $\left.\left|S_{22}\right|\right]$ are shown in Fig. 4(a). In the simulation, the $\mathrm{S}$-parameters are normalized to $50 \Omega$ impedance. Both exhibit two resonances distributed over the desired frequency band. The stand-alone antenna element features an impedance bandwidth of $6.5 \mathrm{GHz}$ (or $23 \%$ ) and $5.70 \mathrm{GHz}$ (or $20 \%$ ) for the vertical and

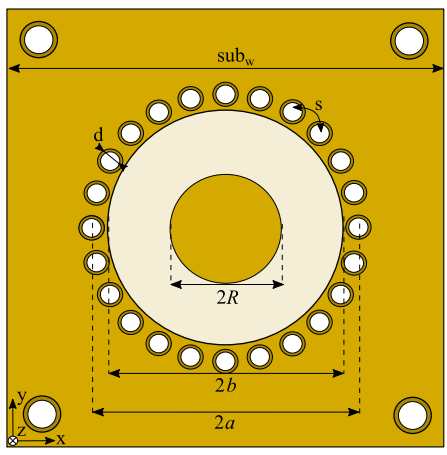

(a)

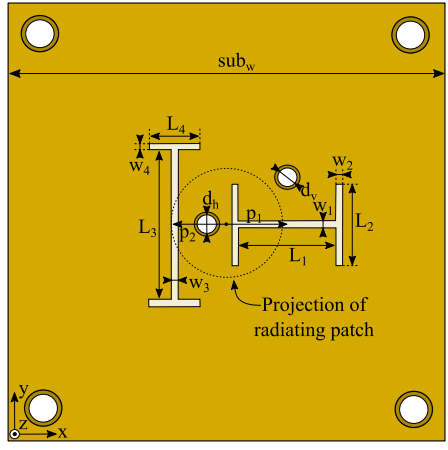

(c)

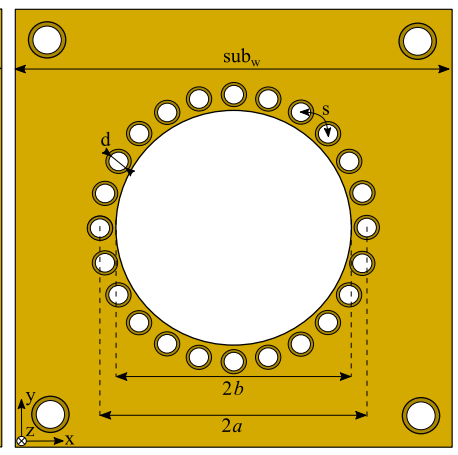

(b)

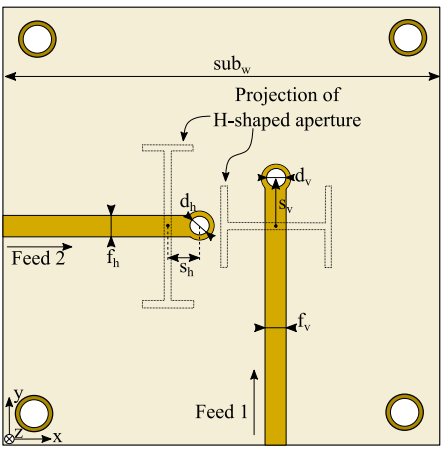

(d)
Fig. 3. Disassembled dual-linearly polarized aperture-coupled AFSIW cavitybacked patch antenna. (a) Bottom view of Layer 1. (b) Bottom view Layer 2. (c) Top view of Layer 3. (d) Bottom view of Layer 3. Optimized dimensions: $s u b_{w}=10.52 \mathrm{~mm}, a=3.2 \mathrm{~mm}, b=2.8 \mathrm{~mm}, R=1.31 \mathrm{~mm}, L_{1}=2.3 \mathrm{~mm}$, $L_{2}=1.9 \mathrm{~mm}, L_{3}=3.4 \mathrm{~mm}, L_{4}=1.2 \mathrm{~mm}, w_{1}=w_{2}=0.15 \mathrm{~mm}$, $w_{3}=w_{4}=0.2 \mathrm{~mm}, p_{1}=1.3 \mathrm{~mm}, p_{2}=1.15 \mathrm{~mm}, f_{v}=f_{h}=0.5 \mathrm{~mm}$, $s_{v}=1.2 \mathrm{~mm}, s_{h}=0.65 \mathrm{~mm}, d_{v}=d_{h}=0.5 \mathrm{~mm}, d=0.6 \mathrm{~mm}, s=0.84 \mathrm{~mm}$, $h_{1}=h_{3}=0.508 \mathrm{~mm}, h_{2}=1 \mathrm{~mm}$. Substrate parameters at $28.0 \mathrm{GHz}:$ RO4350B $\left(\epsilon_{r}=3.66, \tan \delta=0.0037\right)$, FR4 $\left(\epsilon_{r}=4.3, \tan \delta=0.024\right)$.

horizontal polarization, respectively. Moreover, over the entire frequency band of operation, an isolation larger than $25 \mathrm{~dB}$ is guaranteed between both feeds, as shown in Fig. 4(a).

Fig. 4(b) shows the maximum gain of the antenna in the hemisphere along the positive and negative $\mathrm{z}$-axis for each polarization. On average, for both polarizations the gain of the antenna equals $7 \mathrm{dBi}$ and the front-to-back ratio is higher than $17 \mathrm{~dB}$ over the frequency band of interest.

As shown in Fig. 4(c), the total antenna efficiency of the ultra-wideband dual-linearly polarized aperture-coupled AFSIW cavity-backed patch antenna is larger than $85 \%$ over the entire impedance bandwidth, with a maximum of $96 \%$.

\section{Analysis of Antenna Parameters}

One of the key challenges of antenna design at high frequencies is coping with the manufacturing accuracy and tolerances, such as misalignement between layers and variations in antenna dimensions. Manufacturing errors need to be carefully considered from the early design stages to obtain a robust design. Hence, a systematic analysis of the antenna parameters was performed to understand the effects of manufacturing tolerances that are considered relevant for the topology.

In particular, several parameter sweeps were carried out to assess the influence of etching tolerances, misalignment, and 


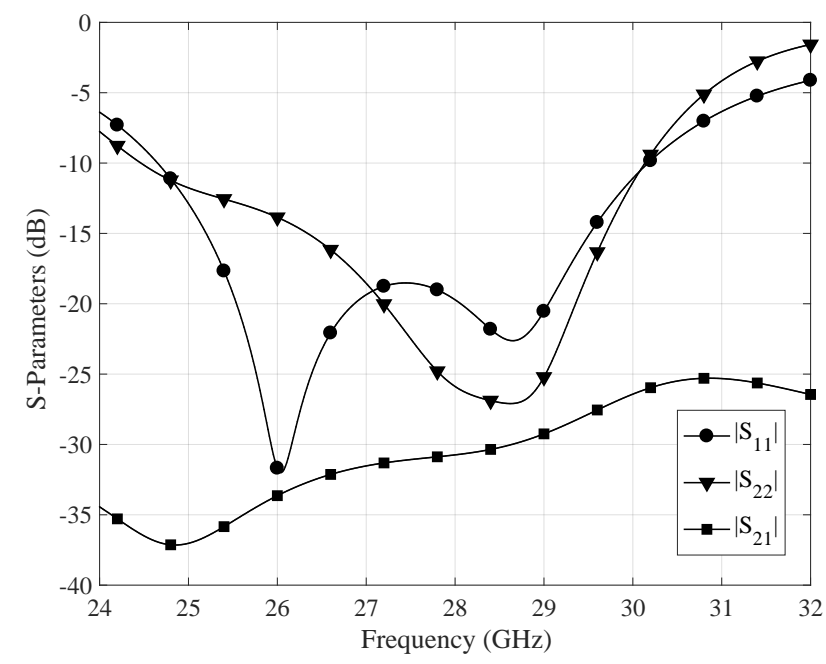

(a)

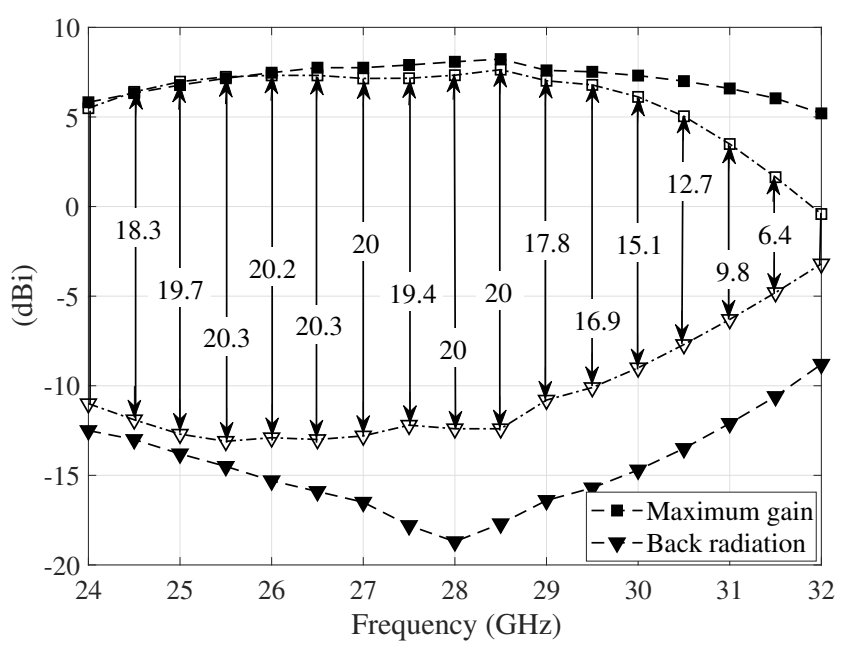

(b)

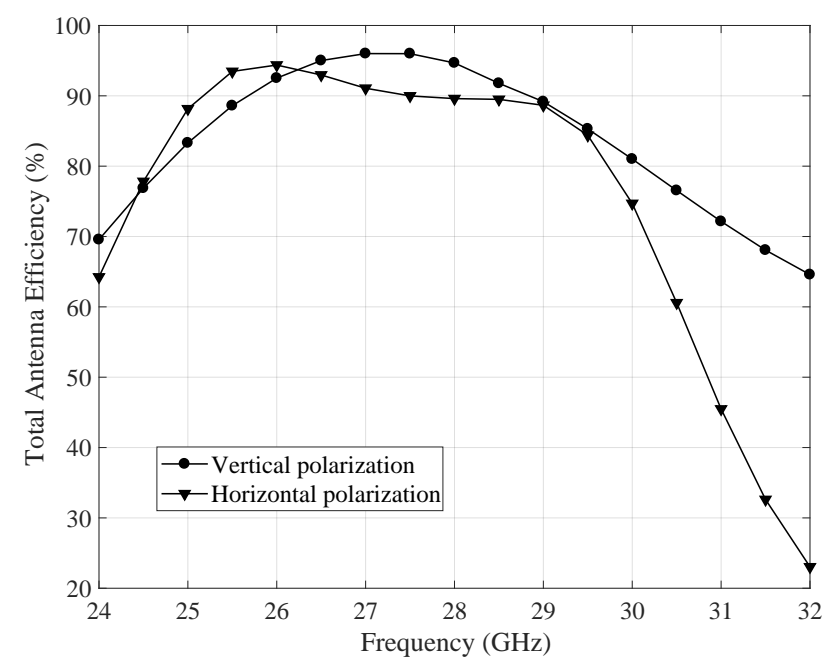

(c)

Fig. 4. Simulated stand-alone wideband dual-linearly polarized aperturecoupled AFSIW cavity-backed patch antenna performance. (a) S-parameters. (b) Maximum gain and back radiation: vertical polarization (dashed line) and horizontal polarizations (dash-dotted line). (c) Total antenna efficieny.

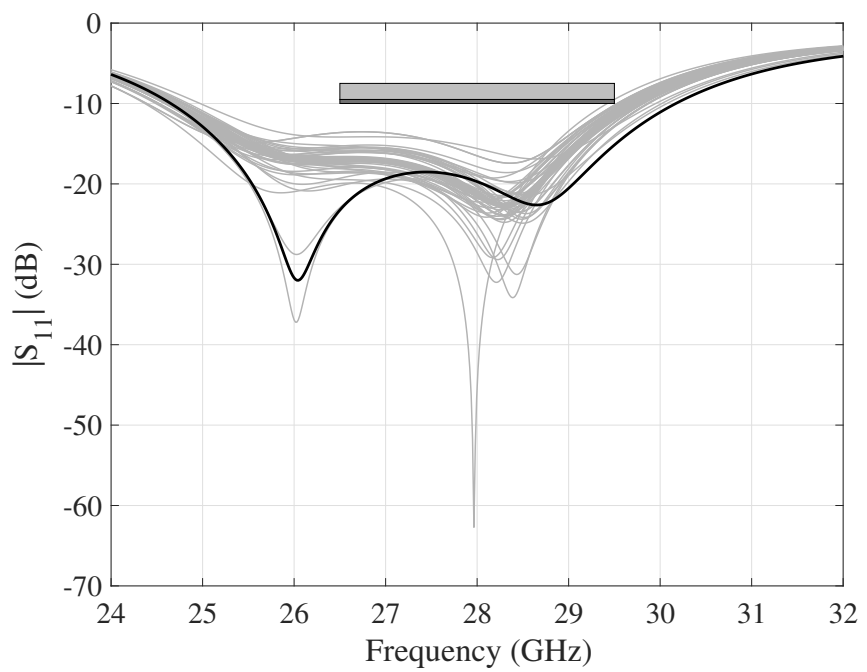

(a)

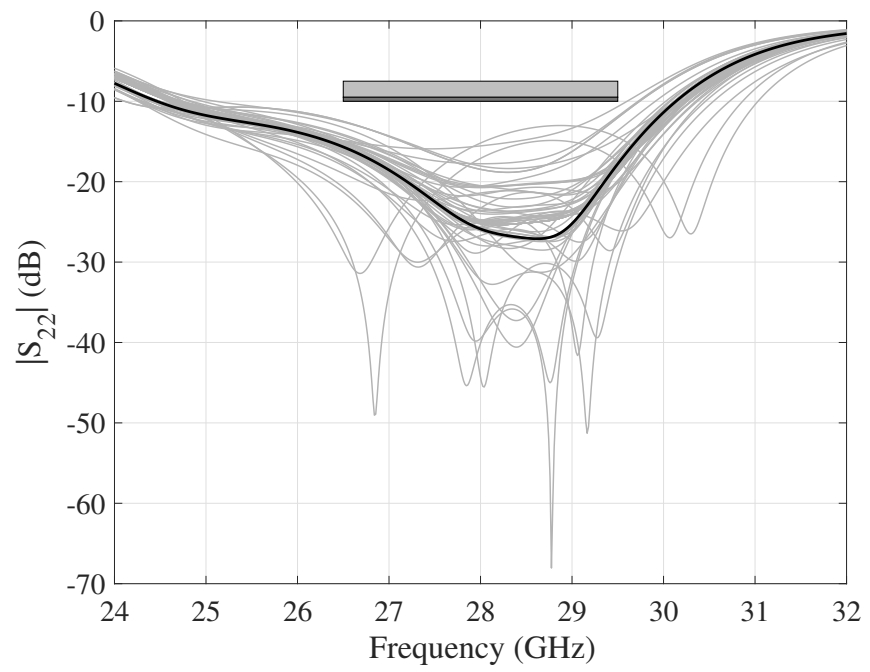

(b)

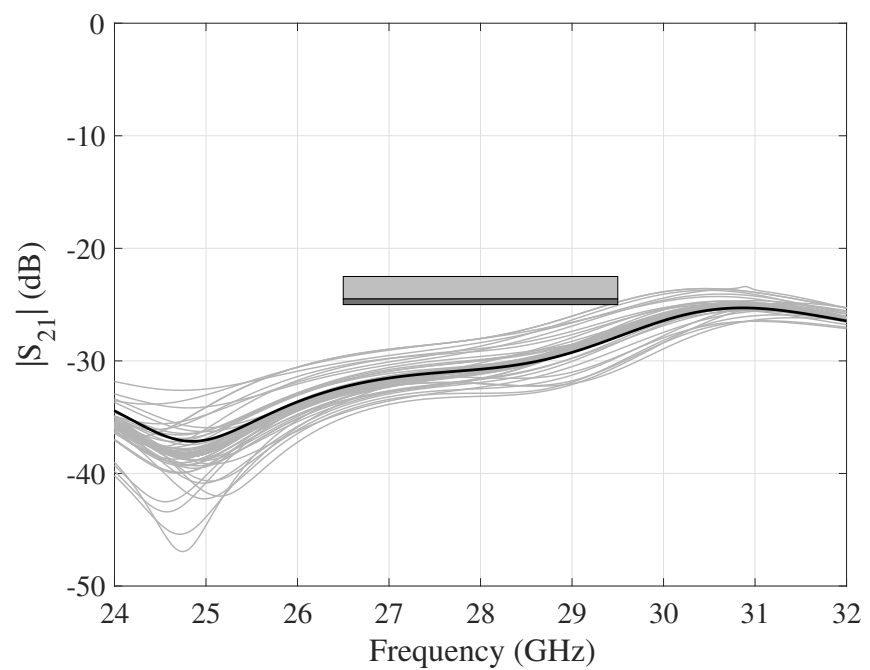

(c)

Fig. 5. Simulated S-parameters for variations on the antenna parameters. Assessing the influence of etching tolerances $[ \pm 25 \mu \mathrm{m}]$, misalignment $[ \pm 100 \mu \mathrm{m}]$, variations in material permittivity $[ \pm 0.1]$, and thickness $[ \pm 50 \mu \mathrm{m}]$ on antenna performance: final design (full black line) and variations (full gray lines). (a) $\left|S_{11}\right|$. (b) $\left|S_{22}\right|$. (c) $\left|S_{21}\right|$. 


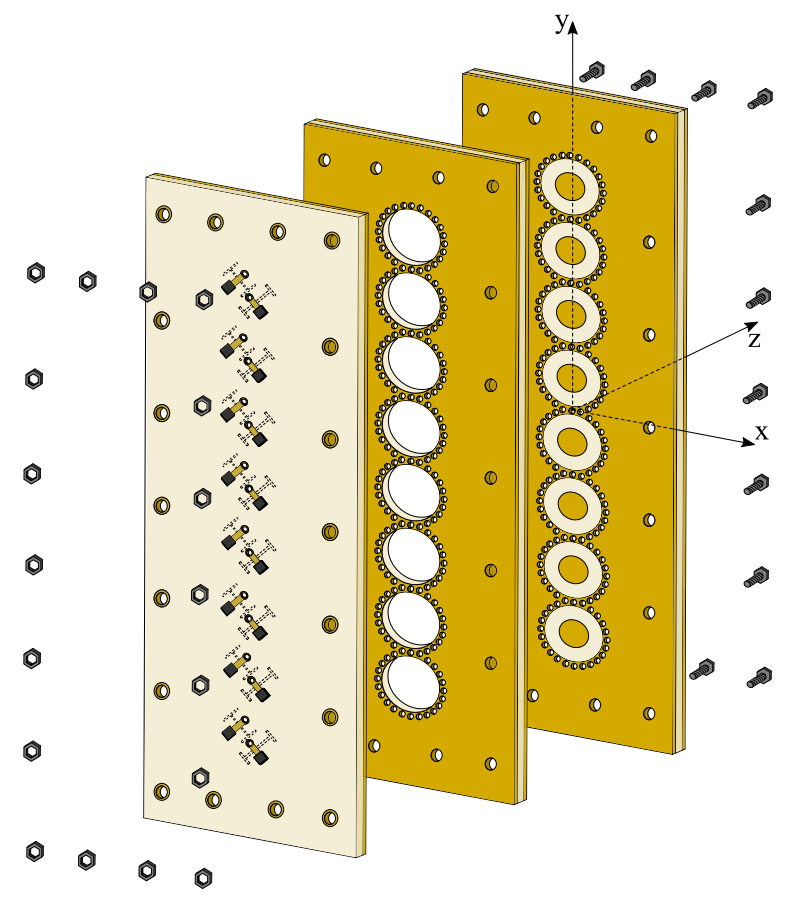

Fig. 6. Exploded view of the eight element aperture-coupled AFSIW cavitybacked patch antenna array.

variations in material parameters (dielectric permittivity and thickness). Worst-case variations in the etching of the different metalizations and in the alignment between the different metalization layers in the assembly are estimated to be $\pm 25 \mu \mathrm{m}$ and $\pm 100 \mu \mathrm{m}$, respectively. The S-parameters were analyzed for misalignments of $\pm 100 \mu \mathrm{m}$ in the $x$ - and $y$-directions, as well as along the first bisection.

Before analyzing the effect of variations in dielectric material parameters, the RO4350B and FR4 laminates' electrical characteristics at $28 \mathrm{GHz}$ were extracted by applying the matrix-pencil two-line method [12]. In the tolerance analysis, a deviation of 0.1 on the dielectric permittivity, being $3 \%$ for RO4350B and $2.3 \%$ for FR4, is considered. Finally, the influence of a dielectric thickness variation of $\pm 50 \mu \mathrm{m}$ on the S-parameters is analyzed.

Fig. 5 displays the simulated S-parameters for all previously discussed parameter sweeps. Based on this extensive parameter analysis, it can be concluded that the proposed design is sufficiently robust against fabrication errors and material variations.

\section{AntEnNA ARRAY}

Fig. 6 shows the exploded view of the proposed linear array. The array comprises eight dual-linearly polarized aperturecoupled AFSIW cavity-backed patch antenna elements. In the array, elements are rotated over $45^{\circ}$ to achieve a higher signalto-noise-ratio [13] and to provide a larger physical space for the compact integration of all required active MMICs.

The proposed array is designed for the frequency range of 26.5 to $29.5 \mathrm{GHz}$. An inter-element spacing of $6.4 \mathrm{~mm}(0.63 \lambda$ at $29.5 \mathrm{GHz}$ ) was chosen to avoid grating lobes when scanning from $-35^{\circ}$ to $+35^{\circ}$. The full array was designed in the frequency domain solver of CST Microwave Studio to validate the active reflection coefficient of the antenna element and to observe overall scanning performance of the array.

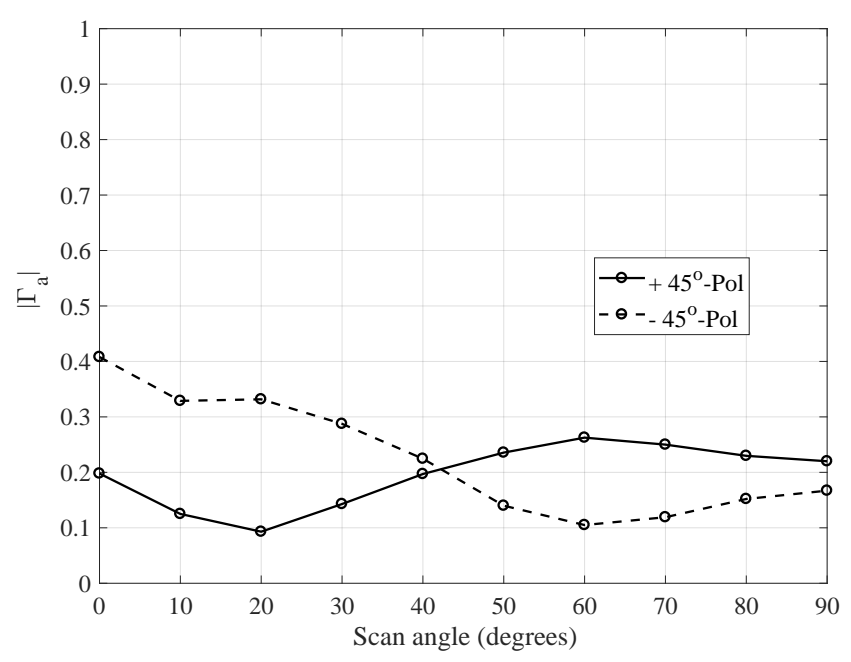

Fig. 7. Simulated active reflection coefficient $\left(\left|\Gamma_{a}\right|\right)$ for the antenna at $28 \mathrm{GHz}$.

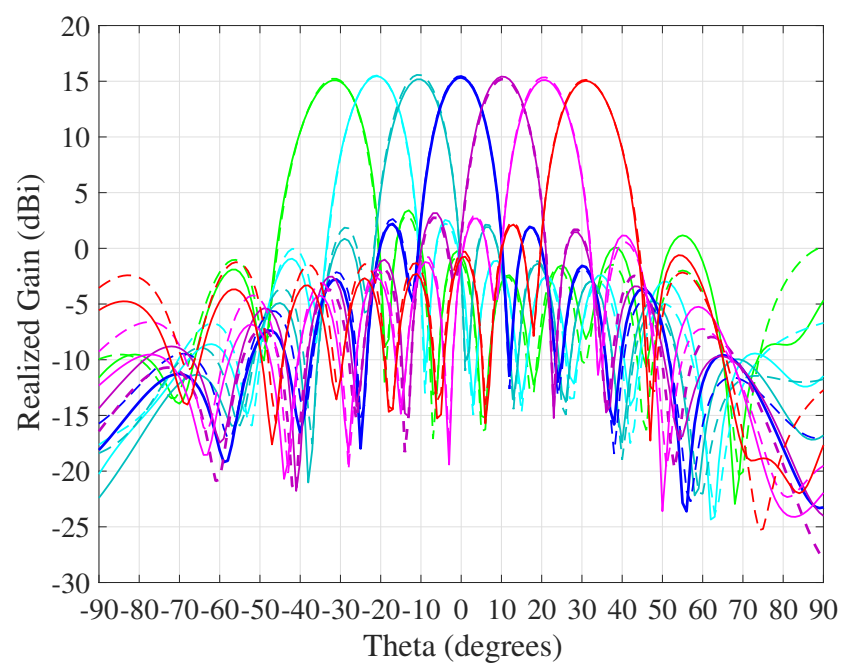

Fig. 8. The simulated radiation patterns of 8 element array at $28 \mathrm{GHz}$ in the YZ-plane. Solid and dashed lines refer to $+45^{\circ}$ and $-45^{\circ}$ polarizations, respectively.

Fig. 7 shows the active reflection coefficient of one of the center antenna elements in the linear array for $\pm 45^{\circ}$ polarizations at $28 \mathrm{GHz}$, when the antenna array is steered in the YZ-plane. No scan blindness was found for both polarizations. For both polarizations, the scanning performance of the array in the YZ-plane was examined. A uniform amplitude distribution is imposed. Fig. 8 shows the radiation patterns at $28 \mathrm{GHz}$ for both polarizations, when scanning in the YZ-plane. A broadside array gain of $15.4 \mathrm{dBi}$ is achieved without gain reduction when the array scans up to $\pm 35^{\circ}$. Moreover, the radiation patterns for both polarizations demonstrate a grating-lobe-free beam scanning range up to a $\pm 35^{\circ}$. The half-power beamwidth amounts to $10.1^{\circ}$ and $77^{\circ}$ in the YZ-plane and XZ-plane, respectively. In this scanning range, the level of the side lobes remain below $-12 \mathrm{~dB}$.

\section{ExPERIMENTAL VALIDATION}

To validate the proposed array topology, a stand-alone antenna element, depicted in Fig. 9, is initially fabricated and validated. In the manufacturing process, the ground plane of 


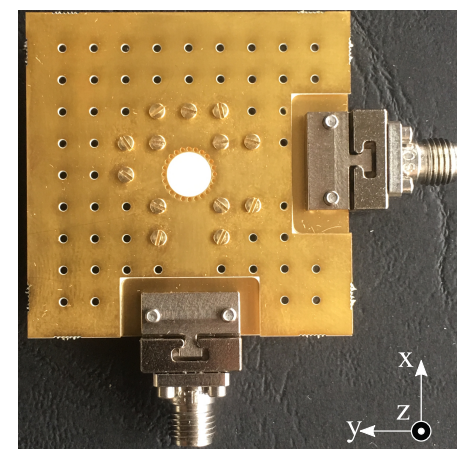

(a)

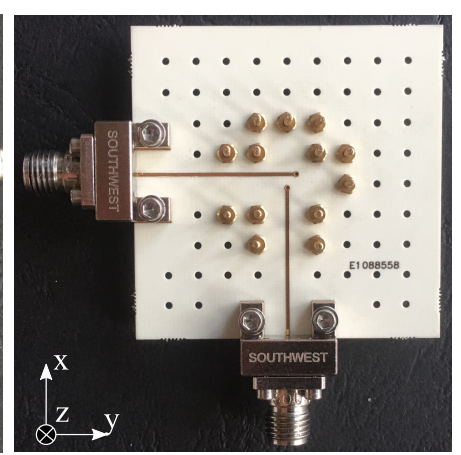

(b)
Fig. 9. Fabricated stand-alone antenna element. (a) Front-side view. (b) Backside view.

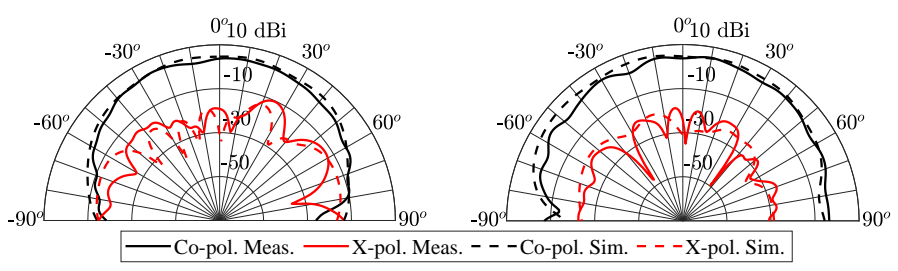

(a)

(b)

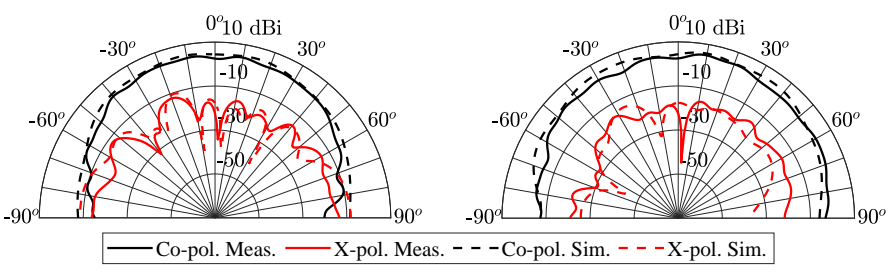

(c)

(d)

Fig. 10. Measured and simulated, including measurement connector, radiation patterns at $28 \mathrm{GHz}$. Vertical polarization: (a) XZ-plane and (b) YZ-plane. Horizontal polarization: (c) YZ-plane and (d) XZ-plane.

the antenna element is extended and an additional row of holes around the cavity is added to reduce the mechanical stress between the layers. Nevertheless, this is merely a precaution and does not have an impact on the antenna performance. The fabricated wideband dual-linearly polarized aperture-coupled AFSIW cavity-backed patch antenna prototype is validated by an Agilent E8364B PNA Microwave Network Analyzer and solder-free End-Launch connectors. The coaxial pins of the connectors are not soldered, but press fit onto the signal trace [see Fig. 9].

The radiation patterns for both orthogonal polarizations are measured in an anechoic chamber by means of an NSI MI near-field antenna measurement system. The measured and simulated gain patterns at $28 \mathrm{GHz}$, both in the XZ- and YZplanes for both polarizations, agree well, as demonstrated in Fig. 10. A broadside gain of $5.6 \mathrm{dBi}$ and a cross polarization of $15 \mathrm{~dB}$ are achieved. For the vertical polarization, the half-power beamwidths in the XZ- and YZ-planes equal approximately $90^{\circ}$ and $111^{\circ}$ whereas the half-power beamwidths approximately amount to $93^{\circ}$ and $80^{\circ}$ in the YZ-plane and XZ-plane for the horizontal polarization.
Furthermore, the complete array will be manufactured and measured to verify the design. The results of these studies will be presented at the conference.

\section{Conclusion}

A new low-cost and low-profile dual-polarized aperturecoupled AFSIW cavity-backed patch antenna topology has been developed as an array element. The antenna covers all worldwide-planned $28 \mathrm{GHz} 5 \mathrm{G}$ bands. Its measured radiation patterns, with a low level of cross-polarization, correspond well with the simulation results. The array element has optimal electrical dimensions to be deployed in a phased antenna array for beamsteering up to $\pm 35^{\circ}$. The active impedance variations with respect to steering angle and scan performance of $8 \times 1$ linear array have been presented. The array exhibits stable radiation patterns within the complete steering range.

\section{REFERENCES}

[1] F. Foglia Manzillo et al., "A multilayer LTCC solution for integrating 5G access point antenna modules," IEEE Transactions on Microwave Theory and Techniques, vol. 64, no. 7, pp. 2272-2283, Jul. 2016.

[2] H. Chu and Y. Guo, "A filtering dual-polarized antenna subarray targeting for base stations in millimeter-wave 5G wireless communications," IEEE Transactions on Components Packaging and Manufacturing Technology, vol. 7, no. 6, pp. 964-973, Jun. 2017.

[3] J. Lota, S. Sun, T. S. Rappaport, and A. Demosthenous, "5G uniform linear arrays with beamforming and spatial multiplexing at 28, 37, 64, and $71 \mathrm{GHz}$ for outdoor urban communication: A two-level approach," IEEE Transactions on Vehicular Technology, vol. 66, no. 11, pp. 99729985, Nov. 2017.

[4] Y. Niu, Y. Li, D. Jin, L. Su, and A. V. Vasilakos, "Survey of millimeterwave communications (mmWave) for 5G: Opportunities and challenges," Wireless Netw., pp. 1-20, Apr. 2015.

[5] G. R. Maccartney, T. S. Rappaport, M. K. Samimi, and S. Sun, "Millimeter-wave omnidirectional path loss data for small cell 5G channel modeling," IEEE Access, vol. 3, pp. 1573-1580, 2015.

[6] C. Mao, S. Gao, and Y. Wang, "Broadband high-gain beam-scanning antenna array for millimeter-wave applications," IEEE Transactions on Antennas and Propagation, vol. 65, no. 9, pp. 4864-4868, Sep. 2017.

[7] J. Lian, Y. Ban, Q. Yang, B. Fu, Z. Yu, and L. Sun, "Planar millimeterwave 2-D beam-scanning multibeam array antenna fed by compact siw beam-forming network," IEEE Transactions on Antennas and Propagation, vol. 66, no. 3, pp. 1299-1310, Mar. 2018.

[8] K. Kibaroglu, M. Sayginer, T. Phelps, and G. M. Rebeiz, "A 64-element $28-\mathrm{GHz}$ phased-array transceiver with $52-\mathrm{dBm}$ EIRP and $8-12-\mathrm{Gb} / \mathrm{s}$ 5G link at 300 meters without any calibration," IEEE Transactions on Microwave Theory and Techniques, vol. 66, no. 12, pp. 5796-5811, Dec. 2018.

[9] W. Hong et al., "Multibeam antenna technologies for 5G wireless communications," IEEE Transactions on Antennas and Propagation, vol. 65, no. 12, pp. 6231-6249, Dec. 2017.

[10] K. Y. Kapusuz, S. Lemey, A. Petrocchi, P. Demeester, D. Schreurs, and H. Rogier, "Polarization reconfigurable air-filled substrate integrated waveguide cavity-backed slot antenna," IEEE Access, 2019.

[11] Eurocircuits. (2015). 'RF Pool'-All the Benefits of Pooling on RF Materials. [Online]. Available: http://www.eurocircuits.com/rf-pool-allthe-benefits-of-pooling-on-rf-materials.

[12] F. Declercq, H. Rogier, and C. Hertleer, "Permittivity and loss tangent characterization for garment antennas based on a new matrix-pencil twoline method," IEEE Trans. Antennas Propag., vol. 56, no. 8, pp. 25482554, Aug. 2008.

[13] Z. N. Chen and K.-M. Luk, Antennas for Base Stations in Wireless Communications. The McGraw-Hill, 2009, ch. 2, pp. 31-94. 